\title{
HLA haplotype A33-B58-Cw10 may modulate radiographic development of bamboo spine in Taiwanese patients with primary ankylosing spondylitis
}

\author{
Ming-Chi Lu ${ }^{\mathrm{a}, \mathrm{b}}$, Kuo-Liang Yang ${ }^{\mathrm{c}}$, Kuang-Yung Huang ${ }^{\mathrm{a}}$, Chien-Hsueh Tung ${ }^{\mathrm{a}}$, Su-Qin Liu ${ }^{\mathrm{a}}$ and \\ Ning-Sheng Lai ${ }^{\text {a,* }}$ \\ ${ }^{a}$ Department of Allergy, Immunology and Rheumatology, Dalin Tzu Chi Buddhist Hospital, Chia Yi, Taiwan \\ ${ }^{\mathrm{b}}$ College of Medicine, Tzu Chi University, Hualien, Taiwan \\ ${ }^{\mathrm{c}}$ Buddhist Tzu Chi Stem Cells Center, Hualien, Taiwan
}

\begin{abstract}
Objective: To evaluate the possible relationship between HLA alleles and bony ankylosis of the spine (bamboo spine) in Taiwanese patients with ankylosing spondylitis (AS).

Methods: A small cohort of HLA-B27 positive AS patients was conducted to analyze the effects of alleles and haplotypes on the development of bamboo spine. DNA typing of HLA class I and class II genes were performed by SSP method on primary ankylosing spondylitis patients with bamboo spine $(n=84)$ and spinal enthesopathy controls $(n=228)$. Odds ratios with 95\% confidence intervals and $P$ value were estimated. Determination of the most probable HLA haplotypes on all patients were constructed by comparison of the alleles carried by each patient with the HLA haplotype database established in Taiwanese population studies using homozygosity approach [1] and by expectation-maximum algorithm [2].

Results: Allele frequencies of HLA A33, B58, Cw10, DR4, DR17 and DQ2 were significantly lower in bamboo patients as compared to non-bamboo controls. In contrast, allele frequencies of A24, B54, Cw15, DR11 and DR14 were significantly higher in bamboo patients. Less remarkably, high frequencies of B39, B51, Cw1 and $\mathrm{Cw} 2$ alleles were also noted in bamboo patients. Considering linkage disequilibria of alleles in haplotypes, HLA-A11-B27-Cw12 was the most common haplotype in both bamboo and non-bamboo groups (95.23\% vs. 91.22\%, respectively, $P=0.238$ ). Haplotypes A33-B58-Cw10, A33-B58-Cw10-DR13 and A33-B58-Cw10-DR17 were significantly lower in bamboo patients as compared to those in controls $(P<0.001, P=0.001$, $P=0.002$, respectively).

Conclusion: Haplotypes A33-B58-Cw10, A33-B58-Cw10-DR13 and A33-B58-Cw10-DR17 showed a strong association with bamboo spine in Taiwanese AS patients. Detection of such haplotypes might be a great aid in the management of patients with the disease.
\end{abstract}

Keywords: HLA, alleles, haplotypes, ankylosing spondylitis, bamboo spine

\footnotetext{
*Corresponding author: Lai Ning-Sheng, MD, PhD, Section of Allergy, Immunology and Rheumatology, Department of Medicine, Dalin Tzu Chi Buddhist Hospital, No2, Ming-Shen Road, Dalin Town, City of ChiaYi, Taiwan. Tel.: +886 52648000 ext 5010; Fax: +886 5 2648555; E-mail: nslai@airpa.org.tw.
}

\section{Introduction}

Spondylopathies are a family of related diseases that include ankylosing spondylitis, reactive arthritis, psoriatic arthropathy, undifferentiated spondylopathy, juvenile chronic arthritis, seronegative oligoarthritis, dactylitis, enthesitis with heel pain, and polyarthritis 
of the lower extremities. The close relationship between AS and HLA antigen B27 is universally recognized $[3,4]$. Several reports have addressed the influence of patient characteristics and HLA alleles on the disease susceptibility and the development of sacroilitis [5], BASDAI index [6], and peripheral arthritis [7]. Although axial arthritis with invariable sacroiliac involvement characterizes the disease, only part of them developed severe, advanced bamboo spine during the disease course. The occurrence of bamboo lesions in patients seems not fully explained by disease duration and may reflect the influence of genetic determinants. Few studies have addressed the genetic susceptibility in the development of bamboo spine. Our previous study had shown an approximate prevalence rate of HLA-B27 in bamboo and spinal enthesopathy patients $(86.2 \%$ vs. $84.7 \%$; data not shown). It might therefore be deduced that alleles other than B27 could be involved in the disease process of spondylophyte formation. The current study further addresses the possible role of HLA class I and class II genes, independent of B27, in the development of bamboo lesions in Taiwanese AS patients.

\section{Patients and methods}

A total of 312 AS patients were enrolled in the current study, including patients with bamboo spine $(N=$ $84)$ and patients with spinal enthesopathy $(N=228)$. All patients fulfilled the modified New York criteria [8]. For characterizing the role of HLA alleles, independent of B27 genes, in the development of bamboo spine, only B27 positive AS patients with at least 10 years duration were included in this study. Patients with clinical AS (without radiographic change), HLA-B27-negative AS, and secondary AS associated with psoriasis, ulcerative colitis, Crohn's disease and Reiter's disease were not enrolled in this study. Radiographs were examined independently by two radiologists blinded to patient identification and without access to clinical or laboratory data. The study was approved by the local IRB of Dalin Tzu Chi General Hospital. Patients were well explained and informed consents were completed before enrollment into the study.

\subsection{DNA typing of HLA-A, $-B,-C,-D R B 1$, and $-D Q B 1$ alleles}

HLA alleles were determined by SSP-DNA typing technique (Dynal ALLSet ${ }^{\mathrm{TM}}$ and CombiSet ${ }^{\mathrm{TM}}$ SSP Kits, DYNAL SCIENCE). Briefly, DNA was obtained from non-heparinized peripheral blood lymphocytes using the QIAamp DNA extraction kit (Qiagen USA). High purity DNA was important and OD 260:280 ratios were required to fall within a range of 1.6-1.8. The polymerase chain reaction (PCR) was carried out in PCR caps containing genomic DNA (50 ng/ $\mu \mathrm{l})$, AmpII Taq DNA polymerase (at $5 \mathrm{IU} / \mu \mathrm{l}$ ) with specific primers and reaction agents. The volumes required were depended on the numbers of PCR reactions (DYNAL SCIENCE). PCR reactions were performed in a PerkinElmer GeneAmp PCR system 9700 with the following protocol: initial denaturation at $96^{\circ} \mathrm{C}$ for $120 \mathrm{~s}$, followed by 10 cycles of $96^{\circ} \mathrm{C}$ for $15 \mathrm{sec}, 65^{\circ} \mathrm{C}$ for $60 \mathrm{se}$ cA20 cycles of $96^{\circ} \mathrm{C}$ for $10 \mathrm{sec} A 61^{\circ} \mathrm{C}$ for $50 \mathrm{sec}$ and $72^{\circ} \mathrm{C}$ for $30 \mathrm{sec}$, ending with $4{ }^{\circ} \mathrm{C}$. The reaction products were analyzed by electrophoresis on $2 \%$ agarose gel.

\subsection{Statistics}

Statistical analysis was performed with SAS software (Version 9.1.3, SAS Institute Inc., Cary, NC, U.S.A.). The Chi-square test or Fisher's exact test was used to analyze categorical proportions and odds ratio. $P$ value $<0.05$ was considered statistically significant.

\subsection{Haplotypes determination of patients}

Determination of the most probable HLA haplotypes on all patients were deduced by comparison of the alleles carried by each patient with the HLA haplotype database established in Taiwanese population studies using homozygosity approach [1] and by expectationmaximum algorithm [2].

\section{Results}

A total of 84 AS patients with bamboo spine and 228 patients with spinal enthesopathy controls were enrolled in this study. The alleles frequency of A33, B58, Cw10, DR4, DR17 and DQ2 were found significantly lower in bamboo patients as compared to the controls $[\mathrm{OR}=0.15,95 \% \mathrm{CI}(0.05-0.44) ; \mathrm{OR}=0.15,95 \% \mathrm{CI}$ $(0.05-0.44) ; \mathrm{OR}=0.29,95 \% \mathrm{CI}(0.20-0.77) ; \mathrm{OR}=$ $0.4795 \%$ CI (0.26-0.87); OR $=0.27,95 \%$ CI $(0.09-$ $0.77) ; \mathrm{OR}=0.24,95 \% \mathrm{CI}(0.08-0.68)$, respectively] (Table 1). In contrast, allele frequencies of A24, B54, Cw15, DR 11 and DR 14 were found significantly higher in bamboo AS patient as compared to the controls [OR $=2.35,95 \% \mathrm{CI}(1.33-4.15)$; OR $=4.58,95 \% \mathrm{CI}(1.88-$ 
Table 1

Frequencies of HLA class I and class II alleles in both AS patient groups

\begin{tabular}{lccccc}
\hline & Bamboo $\%$ & Non-Bamboo \% & OR & $95 \%$ C.I & $P$ \\
\hline A2 & 23.81 & 33.33 & 0.63 & $0.35-1.11$ & 0.106 \\
A11 & 80.95 & 84.21 & 0.80 & $0.42-1.53$ & 0.493 \\
A24 & 33.33 & 17.54 & 2.35 & $1.33-4.15$ & 0.003 \\
A33 & 4.76 & 24.56 & 0.15 & $0.05-0.44$ & $<0.001$ \\
B27 & 100 & 100 & - & - & - \\
B38 & 4.76 & 1.75 & 2.22 & $0.51-15.35$ & 0.136 \\
B39 & 9.52 & 3.50 & 2.89 & $0.91-9.15$ & 0.043 \\
B46 & 4.76 & 12.28 & 0.36 & $0.12-1.05$ & 0.052 \\
B51 & 14.28 & 7.01 & 2.21 & $1.00-4.89$ & 0.046 \\
B54 & 14.28 & 3.50 & 4.58 & $1.80-11.66$ & 0.001 \\
B58 & 4.76 & 24.56 & 0.15 & $0.05-0.44$ & $<0.001$ \\
B60 & 19.04 & 21.05 & 0.88 & $0.47-1.66$ & 0.697 \\
B75 & 4.76 & 5.26 & 0.90 & $0.21-3.08$ & 1.000 \\
Cw1 & 23.81 & 14.03 & 1.91 & $1.02-3.58$ & 0.040 \\
Cw2 & 9.52 & 3.50 & 2.89 & $0.91-9.15$ & 0.043 \\
Cw7 & 28.57 & 24.56 & 1.23 & $0.70-2.15$ & 0.472 \\
Cw8 & 9.52 & 7.01 & 1.39 & $0.57-3.39$ & 0.461 \\
Cw10 & 14.28 & 29.82 & 0.39 & $0.20-0.77$ & 0.005 \\
Cw12 & 90.47 & 92.98 & 0.72 & $0.30-1.74$ & 0.461 \\
Cw14 & 4.76 & 3.50 & 1.38 & $0.29-5.30$ & 0.740 \\
Cw15 & 19.04 & 7.01 & 3.12 & $1.48-6.57$ & 0.002 \\
DR4 & 19.04 & 33.33 & 0.47 & $0.26-0.87$ & 0.014 \\
DR8 & 14.28 & 15.78 & 0.89 & $0.44-1.80$ & 0.744 \\
DR9 & 19.04 & 17.54 & 1.11 & $0.58-2.10$ & 0.759 \\
DR11 & 28.57 & 14.03 & 2.45 & $1.34-4.48$ & 0.003 \\
DR12 & 52.38 & 42.10 & 1.51 & $0.92-2.50$ & 0.106 \\
DR13 & 9.52 & 7.01 & 1.39 & $0.57-3.39$ & 0.461 \\
DR14 & 28.57 & 15.78 & 2.13 & $1.18-3.86$ & 0.011 \\
DR15 & 4.76 & 10.52 & 0.43 & $0.14-1.26$ & 0.114 \\
DR16 & 4.76 & 5.26 & 0.90 & $0.21-3.08$ & 1.000 \\
DR17 & 4.76 & 15.78 & 0.27 & $0.09-0.77$ & 0.010 \\
DQ2 & 4.76 & 17.54 & 0.24 & $0.08-0.68$ & 0.004 \\
DQ4 & 14.28 & 22.80 & 0.56 & $0.28-1.12$ & 0.098 \\
DQ5 & 38.09 & 28.07 & 1.58 & $0.93-2.67$ & 0.089 \\
DQ6 & 23.81 & 28.07 & 0.80 & $0.45-1.43$ & 0.452 \\
DQ7 & 71.42 & 59.65 & 1.69 & $0.98-2.91$ & 0.056 \\
DQ8 & 4.76 & 10.52 & 0.43 & $0.14-1.26$ & 0.114 \\
DQ9 & 19.04 & 17.54 & 2.45 & $1.20-4.99$ & 0.012 \\
\hline & & & & &
\end{tabular}

Table 2

Frequencies of the most common haplotypes in the AS patients with and without bamboo spine

\begin{tabular}{lccccc}
\hline Haplotypes & Bamboo spine $\%$ & Non-bamboo $\%$ & OR & $95 \%$ CI & $P$ value \\
\hline A11/B27/Cw12 & $95.2 \%$ & $91.2 \%$ & 1.92 & $0.64-5.80$ & 0.238 \\
A33/B58/Cw10 & $9.6 \%$ & $40.4 \%$ & 0.16 & $0.07-0.34$ & $<0.001$ \\
A33/B58/Cw10/DR13 or DR17 & $4.8 \%$ & $21.1 \%$ & 0.19 & $0.07-0.54$ & 0.001 \\
A33/B58/Cw10/DR13 or DR17 & $4.8 \%$ & $19.3 \%$ & 0.21 & $0.07-0.60$ & 0.002 \\
\hline
\end{tabular}

O.R. $=$ odds ratio; $95 \%$ C.I. $=95 \%$ confidence interval.

11.66); OR $=3.12,95 \% \mathrm{CI}(1.48-6.57) ; \mathrm{OR}=2.45$, 95\%CI (1.34-4.48), and OR $=2.13,95 \% \mathrm{CI}(1.18-$ 3.86), respectively] (Table 1). A less significant high frequencies of B39, B51, Cw1 and Cw2 alleles were also found in bamboo patients. $(P=0.043,0.046,0.04$ and 0.043 , respectively). Considering linkage disequilibria of the alleles involved in haplotypes, the most common haplotypes in patients were deduced (data not shown) and further analyzed (Table 2). It was found that haplotype A11-B27-Cw12 was the most common haplotypes in both patient groups (95.2\% vs. $91.2 \%$ in bamboo and controls groups, respectively). On the contrary, the haplotypes of A33-B58-Cw10, A33-B58Cw10-DR13 and A33-B58-Cw10-DR17 were significantly lower in bamboo patients $(\mathrm{OR}=0.16,95 \% \mathrm{CI}$ (0.07-0.34); OR =0.19, 95\%CI (0.07-0.54); and OR $=0.21,95 \% \mathrm{CI}(0.07-0.60)$, respectively). 


\section{Discussion}

Ankylosing spondylitis is regarded as a chronic progressive disease leading to a variable degree of restricted mobility of the spine with consequent loss of functional capacity. In a study by Garn et al. [9] on the outcomes of AS in 100 patients with a mean disease duration of 16 years, it was pointed out that radiological manifestations of sacroiliac joints were found in $100 \%$ of AS patients. Spinal changes typical of the AS were found in $58.6 \%$ of patients and final development of "bamboo spine" was found in $18.6 \%$ of patients. In addition, most loss of function occurred during the first 10 years of the disease, and was correlated significantly with spinal X-ray changes of AS and development of "bamboo" spine [9]. Our previous brief study had disclosed that approximately an equal allele frequency of HLA-B27 was found in both bamboo and spinal enthesopathy patients. Likewise, the influence of HLA alleles on patient characteristics and disease severity had been reported in several studies. HLA-DR7 allele had been addressed to be closely related with a more aggressive peripheral joint involvement [5] and the disease onset at younger age in patients with AS [4]. Although having a weak effect on susceptibility to the AS, HLA-DR antigen does not support suggestions that they affect disease severity (BASDAI) [4,10]. In the current brief study, high frequency of haplotype A11-B27Cw12 was present in both patient groups $(95.2 \%$ vs. $91.2 \%$ ) and no significant difference was noted between the two groups. On the contrary, haplotypes A33-B58Cw10, A33-B58-Cw10-DR13 and A33-B58-Cw10DR17 were found significantly lower in bamboo patients group. The data from Tzu Chi Taiwan Marrow Donor Registry (TCTMDR) [11] had previously demonstrated that A33-B58, A11-B27, B58-DR17, B58-DR13, A33-B58-Cw10-DR17, A33-B58-Cw10DR13 and A11-B27-Cw12-DR12 were the rather common haplotypes in Taiwanese. These substantiate the accuracy of the haplotypes A11-B27-Cw12 and A33B58-Cw10 we determined in this study. Since the haplotype A33-B58-Cw10 was found significantly lower in bamboo patients, we postulate that it may play a role in the development of bamboo spine. Although the genes in the HLA loci that we determined in this study were of generic levels which consist of various clusters of subgroups at the allelic levels, however, we trust the haplotype that were involved in modulating the development of bamboo spine is in fact HLA-A*3303$\mathrm{B} * 5801$ due to the exclusive existence and extremely high frequencies of $\mathrm{A} * 3303$ and $\mathrm{B} * 5801$ reported in Taiwanese population studies [7,8].

Homozygosity of B27 has been reported previously to be a risk factor for disease severity in AS. In Taiwan, the prevalence of B27 homozygosity was less than $2 \%$ in AS patients (data not shown). Further data are needed to realize the role of B27 homozygosity in pathogenesis of disease severity of AS including bamboo spine.

In conclusion, development of "bamboo" spine in AS patients may cause major losses of their daily living function and employment potential. Analysis of HLA alleles and haplotypes in AS patients might have a great aid to disease management of the patients.

\section{References}

[1] K.L. Yang and P.Y. Lin, Determination of HLA-A, -B and -DRB1 haplotypes based on allelic homozygosity data in selected bone marrow donors of the Taiwanese marrow donor donor registry, Int J Immunogenet 34 (2007), 385-392.

[2] S.H. Wen, M.J. Lai and K.L. Yang, Human leukocyte antigenA, -B, and -DRB1 haplotypes of cord blood units in the Tzu Chi Taiwan Cord Blood Bank, Hum Immunol 69 (2008), 430436.

[3] D.A. Brewerton, M. Ceffrey, F.D. Hart, D.C.O. Jamas, A. Nicholls and R.D. Sturrock, Ankylosing spondylitis and HL-A 27, Lancet 1 (1973), 904-907.

[4] L. Schlosstein, P.I. Terasaki, R. Bluestone and C.M. Pearson, High association of an HLA antigen, W27, with ankylosing spondylitis, N Engl J Med 288 (1973), 704-706.

[5] F. Berit, S. Anna, J. Virginia et al., The influence of patient characteristics, disease variables and HLA alleles on the development of radiographically evident sacroilitis in juvenile idiopathic arthritis, Arthritis Rheumatism 46 (2002), 986-994

[6] M.A. Brown, L.G. Kennedy, C. Darke et al., The effects of HLA-DR genes on susceptibility to and severity of ankylosing spondylitis, Arthritis Rheumatism 41 (1998), 460-465

[7] R. Sanmarti, M.G. Ercilla, M.A. Brancos, M.C. Cid, A. Collado and J. Rotes-Querol, HLA-class II antigens (DR, DR loci) and peripheral arthritis in ankylosing spondylitis, Ann Rheum Dis 46 (1987), 497-500.

[8] S. Van der Linden, H.A. Valkenburg and A. Cats, Evaluation of diagnosis criteria for ankylosing spondylitis- A proposal for modification of the New York criteria, Arthritis Rheum 27 (1984), 361-368.

[9] J.T. Garn and J.F. Skomsvoll, The outcome of ankylosing spondylitis: a study of 100 patients, British J Rheum 36 (1997), 766-771

[10] G. Varagas-Alarcon, J.D. Londono, G. Hernandez-Pacheco et al., Effects of HLA-B and HLA-DR genes on susceptibility to and severity of spondylopathies in Mexican patients, Ann Rheum Dis 61 (2002), 714-717

[11] C.K. Shaw, T.K. Chang, S.N. Chen and S. Wu, HLA polymorphism and probability of HLA-matched unrelated marrow donors for Chinese in Taiwan, Tissue Antigens 50 (1997), 610-619. 


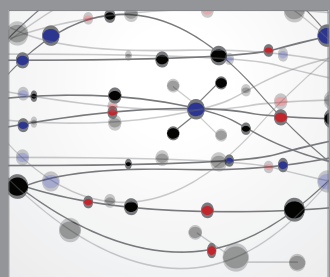

The Scientific World Journal
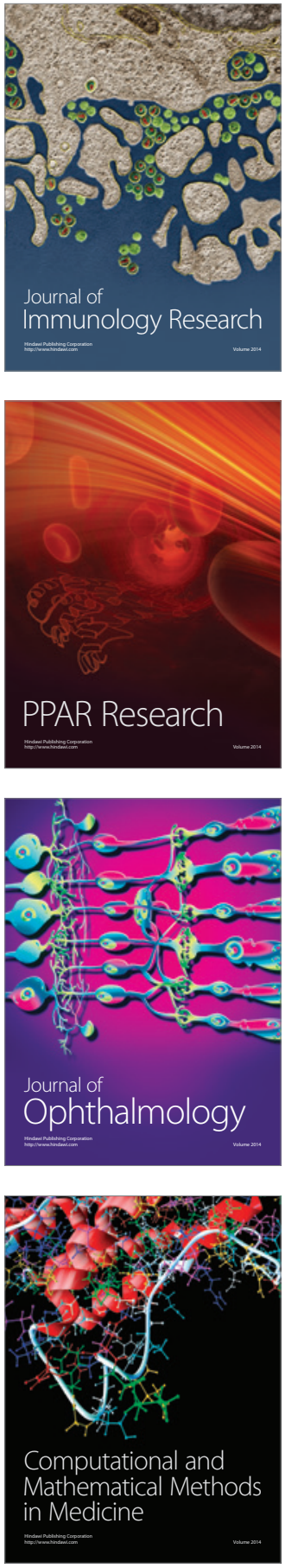

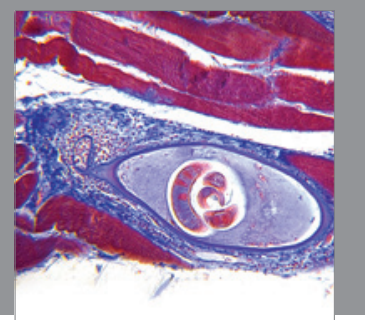

Gastroenterology

Research and Practice
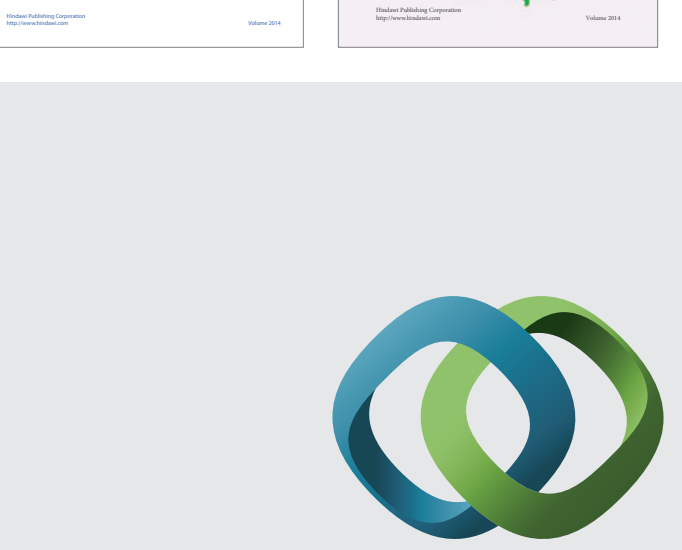

\section{Hindawi}

Submit your manuscripts at

http://www.hindawi.com
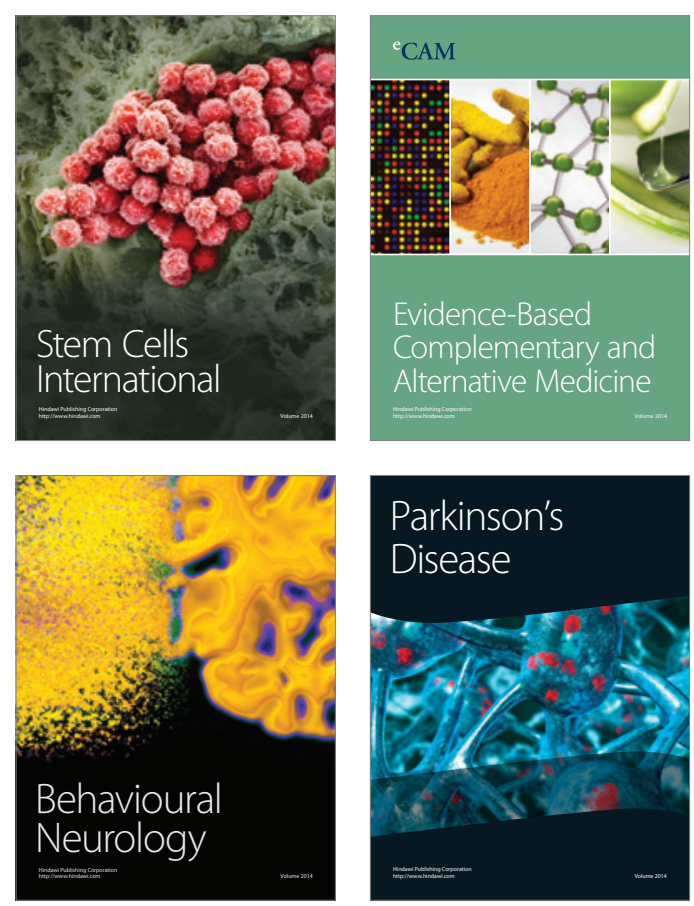

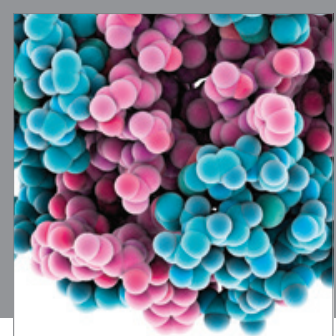

Journal of
Diabetes Research

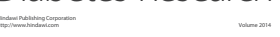

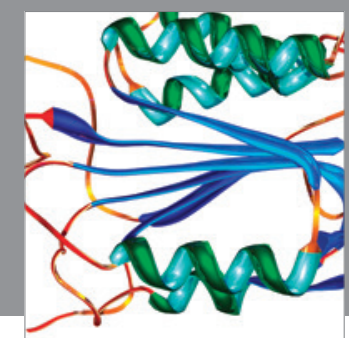

Disease Markers
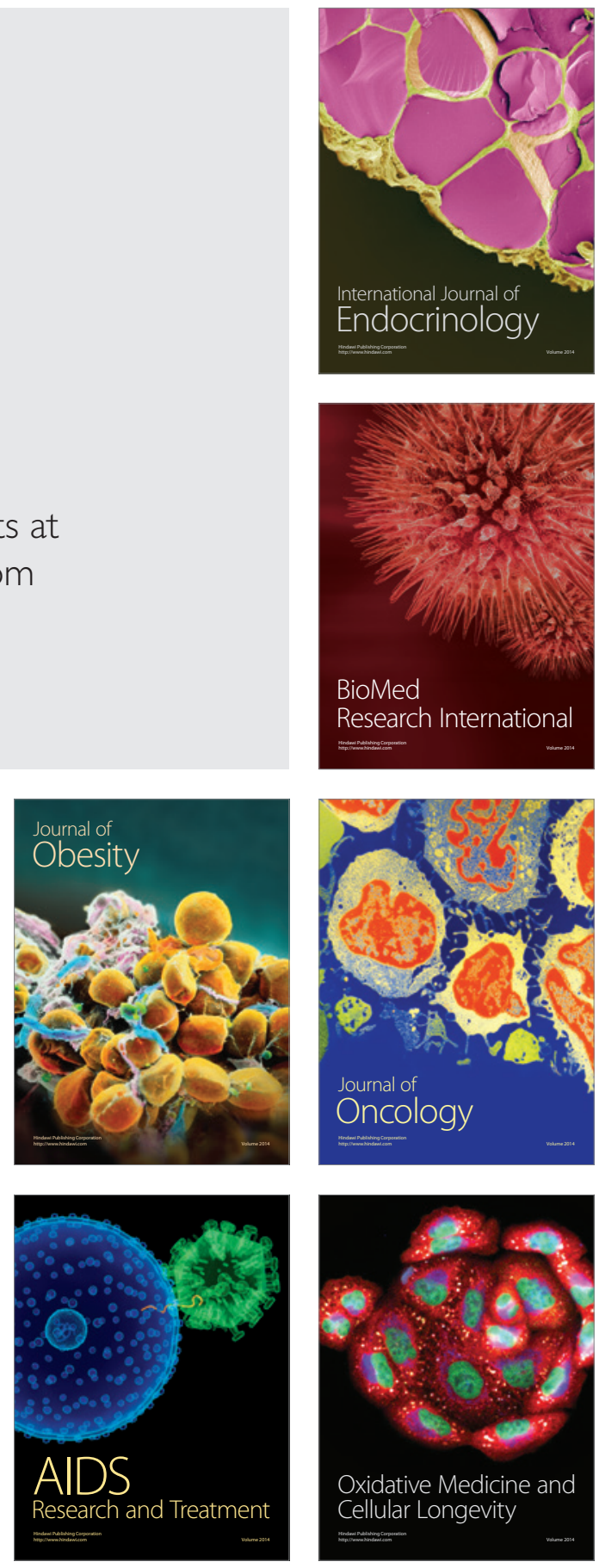